\title{
MODELAGEM MATEMÁTICA COMPUTACIONAL DO FORNO DE UMA UNIDADE DE COQUEAMENTO RETARDADO
}

\author{
A. G. C. ROSAL ${ }^{1}$, M. C. S. CAMELO ${ }^{2}$ e S. LUCENA ${ }^{3}$ \\ ${ }^{1}$ Universidade Federal Rural de Pernambuco, Unidade Acadêmica de Garanhuns \\ ${ }^{2}$ Universidade Federal de Pernambuco, Departamento de Engenharia Química \\ E-mail para contato: deagalindo@gmail.com
}

\begin{abstract}
RESUMO - A unidade de coqueamento retardado é responsável por converter as frações pesadas do petróleo em produtos de maior valor agregado, especialmente, em diesel. $\mathrm{O}$ presente trabalho teve como objetivo o desenvolvimento de um modelo matemático para o forno industrial de craqueamento térmico utilizando um modelo cinético Singh, na caracterização da carga de alimentação por pseudocomponentes e lumps, e modelos para o forno que englobam os balanços de massa e energia, cálculos de propriedades e uso de correlações empíricas para o cálculo de hold-up, perda de carga e equilíbrio termodinâmico.
\end{abstract}

\section{INTRODUÇÃO}

Atualmente, a indústria brasileira de Petróleo e Gás vem investindo em processos de refino capazes de converter frações pesadas de petróleo em derivados mais leves e rentáveis, devido às alterações no perfil de demanda de derivados e a produção crescente de óleos pesados. Dessa forma, tecnologias para processamento de frações residuais de petróleo mostram-se fundamentais para a sobrevivência das refinarias, pois este processamento gera grandes volumes de resíduos na destilação, que consequentemente reduz a rentabilidade da refinaria devido ao seu baixo valor agregado (FILIPAKIS et al., 2008).

A unidade de coqueamento retardado (UCR) é responsável por converter as frações pesadas do petróleo em produtos de maior valor agregado. Nas refinarias brasileiras, este processo vem ganhando destaque, devido à flexibilidade em processar diferentes tipos de carga com menor investimento do que os outros processos de conversão. Geralmente, a unidade de coqueamento é constituída por três equipamentos básicos: a coluna fracionadora, o forno de coqueamento e os tambores de coque (BRASIL, et al., 2011; MOREIRA et al., 2008).

O coqueamento retardado é um processo de craqueamento térmico não catalítico, onde a carga utilizada é geralmente um resíduo de vácuo submetido a condições bastante severas, para produção de produtos líquidos e gasosos mais leves, e um produto sólido (coque de petróleo). No Brasil, a utilização do resíduo atmosférico nessa unidade surgiu como uma adequação do esquema de refino, ocasionado pelo aumento do processamento de óleos pesados (SZKLO; ULLER, 2008). 
O forno de coque destaca-se como o principal equipamento da unidade de coqueamento retardado responsável pelo fornecimento da energia térmica necessária as reações de craqueamento térmico das moléculas de hidrocarbonetos. As reações de craqueamento ocorrem através da associação dos mecanismos via radicais livres e de polimerização das olefinas, sendo a formação de coque justificada por este último (ELLIS; PAUL, 1998).

O presente trabalho teve como objetivo a elaboração de um modelo matemático do forno de coqueamento utilizando-se o modelo cinético de Singh et al. (2005), na caracterização da carga de alimentação por pseudocomponentes e lumps, e modelos para o forno baseados nas equações de balanço de massa e energia desenvolvidas por Sugaya (1994) e Mateus (2008) para um reator do tipo PFR (Plug Flow Reactor) pseudohomogêneo. Além dos cálculos de propriedades e o uso de correlações empíricas para determinação do hold-up, perda de carga e equilíbrio termodinâmico do processo.

\section{METODOLOGIA}

A metodologia utilizada no desenvolvimento da modelagem matemática do forno de coqueamento incluiu a modelagem cinética do processo de craqueamento térmico usado na simulação, a caracterização da carga de alimentação da unidade, e o modelo matemático do forno baseado nas equações de balanço de massa e energia para um reator pseudohomogêneo.

\subsection{Modelagem Cinética do Craqueamento Térmico}

Geralmente, devido à complexidade e ao elevado número de componentes que constituem as frações de petróleo, a caracterização é feita com o menor número de pseudocomponentes que possa representar a mistura de maneira significativa. Na modelagem cinética das reações de craqueamento térmico, os componentes com propriedades semelhantes são agrupados em lumps, onde o fracionamento ocorre de acordo com as faixas de temperatura dos mesmos (DI CARLO; JANIS, 1992).

A representação do sistema reacional é consequentemente melhor quanto maior for a quantidade de lumps, entretanto, a complexidade da modelagem e o número de parâmetros a ser obtidos aumentam consideravelmente (SUGAYA, 1994).

O modelo cinético usado neste trabalho foi proposto por Singh et al. (2005), onde quatro resíduos foram estudados para cinco tempos de residência diferentes entre 3 e 15 minutos e para quatro temperaturas distintas entre 400 e $430^{\circ} \mathrm{C}$. Um sistema reacional com 5 lumps foi desenvolvido por esse autor, como mostra a Figura 1. 


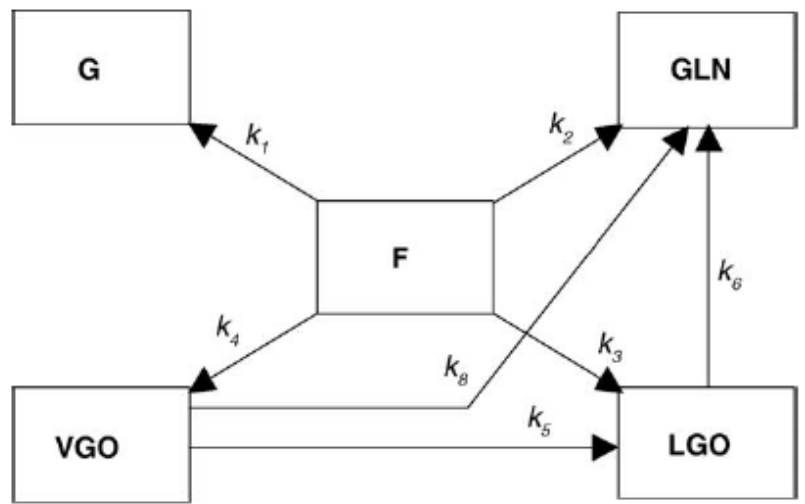

Figura 1 - Modelo cinético de Singh para o craqueamento térmico (SINGH et al., 2005).

Esses modelo foi desenvolvido para um craqueamento térmico de baixa severidade, sendo considerados como lumps o resíduo de vácuo $(\mathrm{F})$, gás $(-\mathrm{C} 5)$, gasolina $\left(\mathrm{PIE}-150^{\circ} \mathrm{C}\right)$, gasóleo leve $\left(150-350^{\circ} \mathrm{C}\right)$ e gasóleo de vácuo $\left(350-500^{\circ} \mathrm{C}\right)$. Inicialmente, o modelo era constituído por 10 parâmetros, mas através dos experimentos e cálculos computacionais percebeu-se que algumas constantes cinéticas poderiam ser descartadas, passando a ser constituído por 5 lumps e 7 parâmetros (SINGH et al., 2005).

Segundo Singh et al. (2005), todas as conversões no craqueamento térmico foram admitidas com cinéticas de primeira ordem, sendo as equações de velocidade escritas pelas Equações 1 a 5.

$$
\begin{aligned}
& \frac{d[F]}{d t}=-\left(k_{1}+k_{2}+k_{3}+k_{4}\right)[F] \\
& \frac{d[V G O]}{d t}=k_{4}[F]-\left(k_{5}+k_{8}+k_{9}\right)[V G O] \\
& \frac{d[L G O]}{d t}=k_{3}[F]+k_{5}[V G O]-\left(k_{6}+k_{10}\right)[L G O] \\
& \frac{d[G L N]}{d t}=k_{2}[F]+k_{6}[L G O]+k_{8}[V G O]-k_{7}[G L N] \\
& \frac{d[G]}{d t}=k_{1}[F]+k_{7}[G L N]+k_{10}[L G O]+k_{9}[V G O]
\end{aligned}
$$

Os valores das constantes cinéticas e os parâmetros para o craqueamento térmico do Resíduo Bombay High são apresentados na Tabela 1. 
Tabela 1 - Parâmetros cinéticos utilizados na simulação do resíduo (SINGH et al., 2005)

\begin{tabular}{c|c|c}
\hline Constante reacional $\left(\mathrm{min}^{-1}\right)$ & Energia de ativação $(\mathrm{E}, \mathrm{kj} / \mathrm{mol})$ & Fator de Frequência $\left(\mathrm{A}_{0}, \mathrm{~min}^{-1}\right)$ \\
\hline $\mathrm{k}_{1}$ & 269,79 & $3,80 \mathrm{E} 17$ \\
$\mathrm{k}_{2}$ & 308,94 & $3,38 \mathrm{E} 20$ \\
$\mathrm{k}_{3}$ & 234,54 & $5,50 \mathrm{E} 15$ \\
$\mathrm{k}_{4}$ & 72,29 & $5,22 \mathrm{E} 3$ \\
$\mathrm{k}_{5,8}$ & 412,50 & $7,80 \mathrm{E} 29$ \\
$\mathrm{k}_{6}$ & 129,29 & $7,66 \mathrm{E} 7$ \\
\hline
\end{tabular}

\subsection{Modelagem do Forno de Coque}

$\mathrm{Na}$ literatura científica existem poucos trabalhos que abordam a modelagem dos fornos de craqueamento térmico de frações pesadas de petróleo, em especial para fornos de coqueamento retardado, onde é necessário o uso de pseudocomponentes e lumps devido à alta complexidade das frações (MATEUS, 2008, SUGAYA, 1994).

Neste trabalho, a modelagem do forno industrial foi baseada no modelo desenvolvido por Sugaya (1994) e Mateus (2008), que consiste nas equações de balanço de massa e energia desenvolvidas para um reator pseudohomogêneo, como mostram as Equações 6 e 7.

$$
\begin{aligned}
& \frac{d C_{350}}{d x}=-\frac{K C_{350} A_{T}\left(h_{L} \rho_{L}+h_{V} \rho_{V}\right)}{G_{T}} \\
& \left(G_{L} C_{P L}+G_{V} C_{P V}\right) \frac{d T}{d x}=-K C_{350} A_{T} h_{L} \Delta H+\pi D U_{T P}\left(T_{w}-T\right)
\end{aligned}
$$

Normalmente, a temperatura nas paredes do tubo usada no balanço térmico possui uma distribuição de temperatura não homogênea no forno de coque, sendo representada pela Equação 8.

$$
K_{w} \frac{d T_{w}}{d x}=\pi D U_{T P}\left(T_{w}-T\right)+Q D \pi d x
$$

Os fornos de coqueamento possuem tubos internos que costuma ser interligados entre si através de cabeçotes mule-ear e curvas em "U". Nos locais onde há presença de acidentes foram assumidos um comprimento equivalente de 50 diâmetros para as curvas em "U" e de 100 diâmetros para os cabeçotes mule-ear, sendo a perda de carga calculada de acordo com as Equações 9 e 10. 
$P=\left(\frac{\Delta P}{\Delta x}\right) d x+\left(\frac{\Delta P}{\Delta x}\right)_{e l}$

$\left(\frac{\Delta P}{\Delta x}\right)_{e l}=\left(h_{L} \rho_{L}+h_{V} \rho_{V}\right) X \sin \theta$

Algumas correlações empíricas usadas no cálculo das propriedades físicas na determinação do hold-up, perda de carga e equilíbrio termodinâmico encontram-se na Tabela 2.

Tabela 2 - Propriedades físicas usadas na modelagem

\begin{tabular}{l|c|c}
\hline \multicolumn{1}{c|}{ Propriedade } & Fase Líquida & Fase Vapor \\
\hline Densidade & Hankinson-Thomson (1979) & SRK \\
Viscosidade & Kendall-Monroe(1917) & Dean-Stiel (1965) \\
Condutividade Térmica & API TDB (1997)-12A2.1 & API TDB (1997) - 12B2.1 \\
Capacidade Calorífica & API TDB (1997) - 7D2.2 & API TDB (1997) - \\
\hline
\end{tabular}

\section{RESULTADOS}

A caracterização do óleo e a obtenção de algumas propriedades usadas na simulação do forno de coqueamento foram obtidas através da utilização do HYSYS, onde uma curva TBP para o óleo foi calculada com os dados disponíveis na carta de óleo Frade da Chevron. A partir desta curva TBP foram divididos os cortes de interesse na modelagem cinética, sendo obtidos os valores de diversas propriedades para os lumps, que são apresentados na Tabela 3.

Tabela 3 - Propriedades dos lumps obtidas através da curva TBP

\begin{tabular}{l|c|c|c|c|c|c}
\hline Componente & $\begin{array}{c}\text { Faixas do } \\
\text { Corte }\end{array}$ & $\begin{array}{c}\text { NBP } \\
\left({ }^{\circ} \mathbf{C}\right)\end{array}$ & $\begin{array}{c}\text { Massa } \\
\text { Molar } \\
(\mathbf{g} / \mathbf{m o l})\end{array}$ & $\begin{array}{c}\text { Densidade } \\
\left(\mathbf{k g} / \mathbf{m}^{\mathbf{3}}\right)\end{array}$ & $\begin{array}{c}\text { Viscosidade } \\
\text { líquido a } \\
\mathbf{1 0 0}^{\mathbf{}} \mathbf{F}(\mathbf{c P})\end{array}$ & $\begin{array}{c}\text { Viscosidade } \\
\text { líquido a } \\
\mathbf{2 1 0} \mathbf{F}(\mathbf{c P})\end{array}$ \\
\hline Gás & até $12,8^{\circ} \mathrm{C}$ & 11,8 & 61,2 & 606,6 & 0,196 & 0,108 \\
Gasolina & $12,8-150^{\circ}$ & 113,1 & 87,7 & 702,9 & 0,316 & 0,197 \\
Gasóleo Leve & $150-350^{\circ} \mathrm{C}$ & 279,4 & 218,3 & 834,7 & 2,582 & 0,952 \\
Gasóleo Pesado & $350-500^{\circ} \mathrm{C}$ & 421,8 & 379,4 & 895,3 & 28,703 & 4,259 \\
Resíduo & $500^{\circ} \mathrm{C}+$ & 605,9 & 648,5 & 958,2 & 5463,809 & 84,144 \\
\hline
\end{tabular}


O desenvolvimento do código computacional para a simulação do forno foi realizado em ambiente Matlab ${ }^{\circledR}$, e utilizou o método das diferenças finitas para resolver o conjunto de equações diferencias ordinárias do modelo. As simulações computacionais foram realizadas para predizer os perfis de temperatura, pressão, assim como, os perfis referentes às frações mássicas dos lumps estabelecidos pelo modelo e a fração vaporizada no equipamento como mostram as Figuras 2 a 4.

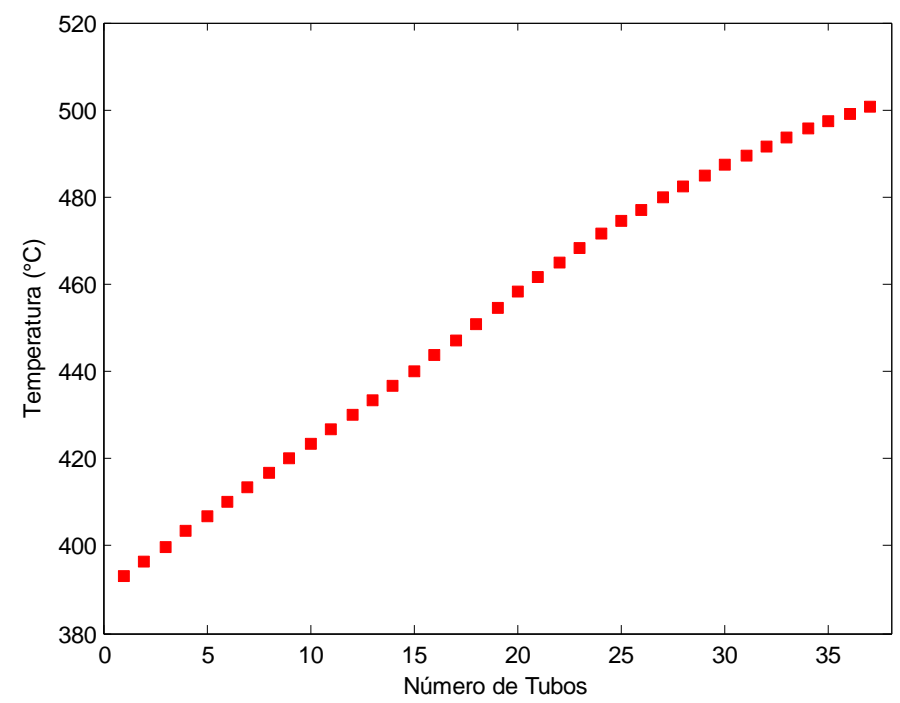

Figura 2 - Perfil de temperatura ao longo dos tubos no forno.

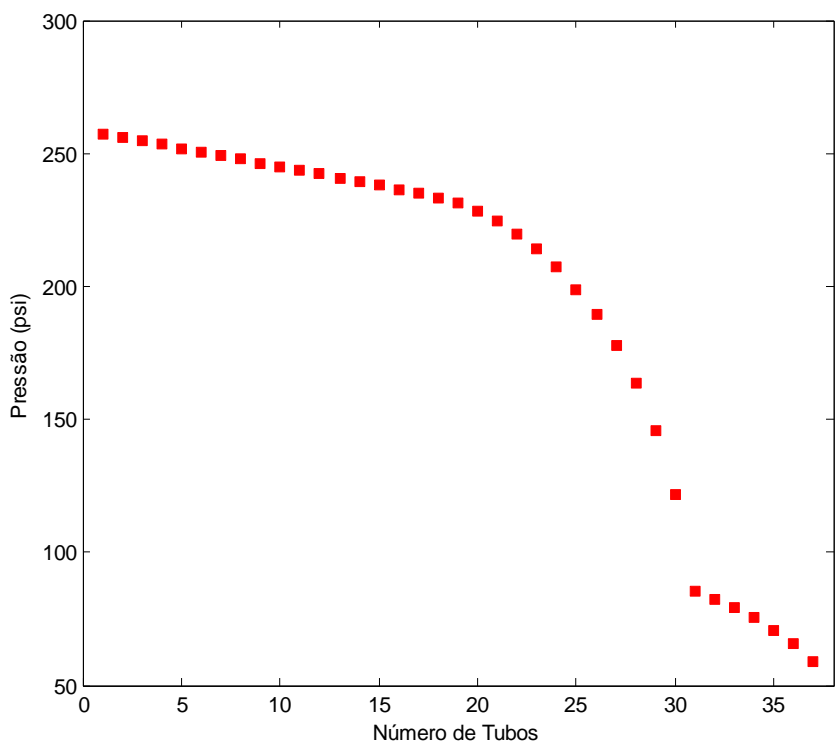

Figura 3 - Queda de pressão ao longo dos tubos no forno. 


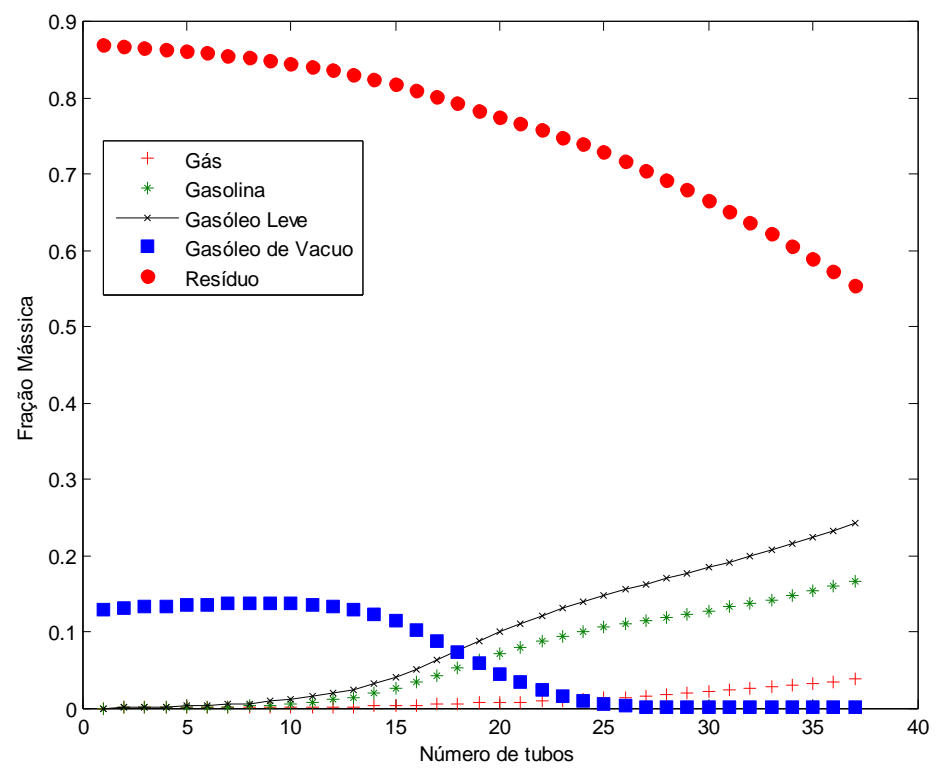

Figura 4 - Fração mássica dos lumps ao longo dos tubos no forno.

O modelo matemático desenvolvido no presente trabalho mostrou uma concordância muito significativa quando comparado os valores de temperatura, pressão ao longo dos tubos do forno com o modelo de Mateus (2008). Uma característica que deve ser ressaltada, é que a cinética utilizada é valida para uma faixa de temperatura mais baixa, fato que leva a uma produção maior de leves do que o modelo produzido pelo Mateus (2008) o que ocasiona em uma maior vaporização que consequente influencia no aumento de velocidade média das fases ao longo dos tubos. A cinética de craqueamento térmico de Singh et al. (2005) favorece a conversão da carga em produtos leves, principalmente gasóleo leve e gasolina, a partir do tubo 12, que atinge um valor máximo na saída do reator em gás $4 \%$, gasolina $18 \%$ e gasóleo leve $25 \%$.

A comparação dos resultados obtidos na modelagem matemática com o forno industrial 23 H-1 da Refinaria Presidente Bernardes (Petrobras) é apresentado na Tabela 4.

Tabela 4 - Comparação entre os resultados obtidos pela modelagem com forno industrial da Petrobras.

\begin{tabular}{l|c|c}
\hline Parâmetro & Modelo Desenvolvido & Forno 23 H-1 \\
\hline $\mathrm{T}_{\text {saída }}\left({ }^{\circ} \mathrm{C}\right)$ & 500,64 & 500 \\
$\mathrm{P}_{\text {saída }}(\mathrm{psi})$ & 59,29 & 65 \\
\hline
\end{tabular}




\section{CONCLUSÕES}

O modelo matemático desenvolvido se apresentou como uma ferramenta adequada para prever a distribuição dos produtos obtidos para o processo de coqueamento retardado. Apresentou vantagens em relação aos modelos desenvolvidos por Mateus (2008) e Sugaya (1994) devido à proposta de utilização de modelos cinéticos com redução de lumps, necessitando assim de uma demanda menor parâmetros cinéticos e, consequentemente, de menos experimentos na adaptação da modelagem para diferentes tipos de carga. O modelo também se apresentou viável quanto a previsão das condições operacionais do equipamento como a queda de pressão e temperatura ao longo da tubulação do forno.

\section{REFERENNCIAS}

BRASIL, N. I do; ARAÚJO, M. A. S.; DE SOUSA, E. C. M. Processamento de Petróleo e Gás: petróleo e seus derivados, processamento primário, processos de refino, petroquímica e meio ambiente. Rio de Janeiro: LTC, 2011.

DI CARLO, S., JANIS, B. Composition and visbreakability of petroleum residues. Chemical Engineering Science, 47, 2695-2700, 1992.

ELLIS, P.J., PAUL, C.A. Tutorial: Delayed Coking Fundamentals. AIChE 1998, Spring National Meeting, New Orleans-LA, 1998.

FILIPAKIS, S.D., SILVA, M.S.A.J., GUIMARÃES, R.C.L. Estimativa da qualidade e dos rendimentos de produtos de coqueamento a partir do processamento de petróleos nacionais. In: Rio Oil \& Gas Expo and Conference, 2008.

MOREIRA, F.S., SEIDL, P.R., GUIMARÃES, M.J.O.C. A importância das novas unidades de coqueamento retardado no mercado de combustíveis. In: Rio Oil \& Gas Expo and Conference, 2008.

SINGH, J., KUMAR, M.M., SAXENA, A.K., KUMAR, S.. Reaction pathways and product yields in mild thermal cracking of vacuum residues: A multi-lump kinetic model. Chemical Engineering Journal, 108, 239-248, 2005.

SUGAYA, M. F. Cinética e modelagem do craqueamento térmico de frações residuais do petróleo. Dissertação (Mestrado em Engenharia Química) - Faculdade de Engenharia Química, Universidade Estadual de Campinas. 1994.

SZKLO, A. S., ULLER, V. C. Fundamentos do refino de petróleo: tecnologia e economia. $2^{\mathrm{a}}$ ed. rev. Rio de Janeiro: Interciência, 2008. 